\title{
Synthesis of Polypyrrole Using Ammonium Peroxy Disulfate (APS) as Oxidant Together with Some Dopants for Use in Gas Sensors
}

\author{
Hemant K. Chitte ${ }^{1}$, Narendra V. Bhat ${ }^{2}$, Ajit V. Gore ${ }^{2}$, Ganesh N. Shind ${ }^{3}$ \\ ${ }^{1}$ Dnyansadhana College, Near Eternity Mall, Thane, India; ${ }^{2}$ Bombay Textile Research Association, LBS Marg, Mumbai, India; \\ ${ }^{3}$ Indira Gandhi College, Cidco Colony, New Nanded, India. \\ Email: hkchitte@yahoo.co.in
}

Received June $3^{\text {rd }}, 2011$; revised June $24^{\text {th }}, 2011$; accepted July $25^{\text {th }}, 2011$.

\begin{abstract}
Polypyrrole (Ppy) was synthesized using Ammonium Peroxy Disulfate (APS) as oxidant in a standard ratio of monomer to oxidants at $5{ }^{\circ} \mathrm{C}$. Attempts were made to increase the electrical conductivity by using various dopants viz. Lithium per Chlorate $\left(\mathrm{LiClO}_{4}\right)$, para-Toluene Sulfonate ( $\left.p-T S\right)$ and Napthalene Sulfonic acid (NSA). The materials were characterized using FTIR, X Ray diffraction and SEM. The electrical conductivity was measured by two probe method and was found to be in the range of $10^{-3} \mathrm{~S} / \mathrm{cm}$. Thin films of these preparations were casted on the interdigited electrodes to study the detection of gas such as ammonia. It was found that for the pure Ppy when ammonia gas was allowed to flow in, there was a sudden increase in the current, which decreased rapidly when gas was stopped. However when Ppy doped with p-TS, NSA and $\mathrm{LiClO}_{4}$, the trend was reversed.
\end{abstract}

Keywords: Polypyrrole, Structure, Doping, Gas Sensor, Ammonia

\section{Introduction}

Over the last few decades, conducting polymers have emerged as a new class of materials with interesting electron-transport behavior and a material with immense potential in technological applications. Their ease of processing together with their chemically tunable properties makes them especially useful in electronic, optoelectronic and electromechanical devices [1-5]. One such area where the conducting polymers have shown great promise is in sensory applications. Delocalized electronic states combined with the restriction on the extent of delocalization makes most of the conductive polymers behave like p-type semiconductors. As these polymers are Redox-active, their conductivity can be changed by means of doping/dedoping. A great number of sensing applications are designed by exploiting the very nature of conducting polymers [6].

Conducting polymers, such as polypyrrole (Ppy), polyaniline (Pani), polythiophene (PTh) and their derivatives, have been used as the active layers of gas sensors since early 1980s [7]. In comparison with most of the commercially available sensors, based usually on metal ox- ides and operated at high temperatures, the sensors made of conducting polymers have many improved characteristics. They have high sensitivities and short response time; especially, these features are ensured at room temperature. Conducting polymers are easy to be synthesized through chemical or electrochemical processes, and their molecular chain structure can be modified conveniently by copolymerization or structural derivations [8]. Polypyrrole, in the form of films, has been used for sensors for detection of various gases and volatile organic compounds [9-13]. Polypyrrole sensors are sensitive with good response to these gases, showing larger response to polar than non polar compounds.

Ammonia is a toxic gas even at low concentrations. Environmental and health safety restrictions or recommendations determine a very low allowable concentration, which has not been reliably detectable with the commercially available sensors. These are suffering from the lack of high sensitivity and selectivity. Generally they also need heating for their operation. All industries where ammonia is produced or used or is a by-product, as well as agricultural plants where ammonia is present, its early detection is highly needed [14]. 
The physical properties of conducting polymers strongly depend on the type of dopants and the doping levels. The doping levels can be easily changed by chemical reactions at room temperatures and this provides a simple technique to facilitate detection of several gases. Most of the conducting polymers are doped/undoped by redox reactions. When such conducting polymeric material is brought in contact with either a gas or a liquid, transfer of electrons from or to the analyte takes place. Electron transferring can cause the change in resistance and work function of the sensing material. When Ppy is exposed to some gases, redox reaction can take place. Gases such as $\mathrm{NO}_{2}$ and $\mathrm{I}_{2}$ which are electron acceptors can remove electrons from the aromatic ring of polypyrrole. When this occurs, for a p-type conductive polymer, the doping level as well as electric conductance of the conductive polymers is enhanced. On the other hand an electron donating gas, such as ammonia, reacts with the Ppy, the electrical conductance falls down sharply. However when desorption of the gas occurs the process can be reversed. The following reaction is involved in the process [15].

$$
\begin{array}{ll}
\mathrm{Ppy}+\mathrm{NH}_{3} \rightarrow \mathrm{Ppy}+\mathrm{NH}_{3}^{2+} & \text { absorption } \\
\mathrm{Ppy}+\ddot{\mathrm{N}} \mathrm{H}_{3} \rightarrow \mathrm{Ppy}^{+}+\ddot{\mathrm{NH}}_{3} & \text { desorption }
\end{array}
$$

In spite of such advances, the results obtained for many gases may differ from this generalized postulate of detection mechanism. For example work reported in thin polycarboazole film sensors shows increase in conductance when the electron donating gas has been used [16].

Conducting polymers usually can be synthesized by chemical or electrochemical techniques, oxidizing the corresponding monomers. Chemical oxidation involves mixing monomer and oxidant in solution. The most widely used oxidants are Ammonium Peroxy Disulfate, Ferric Chloride, Hydrogen peroxide, Potassium dichromate, Cerium sulfate, and so on. Both aqueous and organic media are used.

In the present paper we report preparation of polypyrrole in pure form and by using dopants such as $\mathrm{LiClO}_{4}$, p-TS and NSA. The structure and morphology of these materials has been investigated using the methods of FTIR, X-Ray diffraction and SEM. The electrical conductivity has been measured by two probe method. The response of these materials, when exposed to ammonia is being reported.

\section{Experimental Methods and Preparations}

Pyrrole (Sisco Research laboratory, 99\% pure) was distilled before use. All other reagents and solvents obtained from SDL were of reagent grade and were used as received. All solutions were prepared using distilled water.
All reactions were conducted at a temperature of $5^{\circ} \mathrm{C}$ [17]. The solution of the oxidizing agent, APS, was prepared using distilled water and was used in the ratio of 1:2.4 (monomer:oxidant) [12]. Dopants were mixed with Pyrrole solution $(10 \% \mathrm{w} / \mathrm{w})$ and stirred for 30 minutes for proper mixing and then the oxidant solution was added slowly.

The Polypyrrole was prepared by chemical polymerization method. $1 \mathrm{M}$ Pyrrole solution was prepared using distilled water and then mixed with an oxidizing agents in the ratio mentioned above, slowly under constant stirring for 30 minutes. Then the polymerization was conducted for 4 hours under constant stirring. This preparation was kept unagitated for 24 hours so that Ppy powder settled down. The Polypyrrole powder was filtered out under vacuum and washed with distilled water several times to remove any impurities present. The Polypyrrole was dried for 2 days at room temperature.

The chemical bonding was analyzed using FTIR spectroscopy by FTIR spectrometer model Perkin-Elmer Ltd. system 2000 using $\mathrm{KBr}$ pellets. The Polypyrrole was characterized by XRD using PAnalytical (Philips), model XpertPro. The XRD patterns were recorded between $2 \theta$ $=10^{\circ}$ to $40^{\circ}$ The $\mathrm{X}$-ray diffractometer uses $\mathrm{CuK} \alpha$ radiation of $\lambda=1.5418 \AA$ generated at $40 \mathrm{kV} / 20 \mathrm{ma}$.

The morphology of Polypyrrole was examined using Scanning Electron Microscope (SEM) JEOL make JSM5400 model. The Polypyrrole was tested for conductivity by preparing its pellets of area of cross section of $1 \mathrm{sq} \cdot \mathrm{cm}$. and $1 \mathrm{~mm}$. thickness. The conductivity was tested by two probe apparatus fabricated in our laboratory. It consists of a copper plate which serves as one electrode, heated from the bottom to raise the temperature. The second electrode was a copper rod which was spring loaded to give enough pressure on the sample pallet so that electrical contact is maintained The conductivity was measured at room temperature and various temperatures up to 70 ${ }^{\circ} \mathrm{C}$ in the voltage range from 0 to $12 \mathrm{~V}$.

In order to measure the gas response, interdigited electrodes, separated by $1 \mathrm{~mm}$. from all sides, were prepared on a printed circuit board (PCB) and the slurry of Polypyrrole powder prepared with distilled water was spread over it uniformly and dried for 24 hours under vacuum at room temperature. A specially prepared gas chamber was used in which the PCB was fitted firmly. Various gases were passed through the chamber at room temperature with Nitrogen gas as a carrier. The current was recorded, at a constant voltage, for every 15 seconds for the total time of 3 minutes. The flow of the gas was stopped and the desorption was also recorded for interval of every 10 seconds till current recovered up to $90 \%$ of its original value. The gas response was recorded con- 
tinuously for 3 cycles.

\section{Result and Discussions}

\subsection{Characterization of Ppy}

\subsubsection{FTIR Spectroscopy}

The Polypyrrole powders prepared in different ways were analyzed by FTIR. FTIR spectra showed the main characteristic peaks at $1558 \mathrm{~cm}^{-1}, 1542 \mathrm{~cm}^{-1}$ and 1471 $\mathrm{cm}^{-1}$ corresponding to the fundamental vibrations of polypyrrole ring. The band at $1294 \mathrm{~cm}^{-1}$ corresponds $\mathrm{C}-\mathrm{H}$ deformation. Other low intensity peaks are observed at around $2927 \mathrm{~cm}^{-1}-2814 \mathrm{~cm}^{-1}$ which can be attributed to aromatic $\mathrm{C}-\mathrm{H}$ stretching vibrations. The peaks at 1685 $\mathrm{m}^{-1}$ and $801 \mathrm{~cm}^{-1}$ represents $\mathrm{C}=\mathrm{N}$ and $\mathrm{C}-\mathrm{N}$ bonds, the bond of $\mathrm{C}-\mathrm{H}$ in plane deformation vibration is situated at $985 \mathrm{~cm}^{-1}$ and of the C-C out of plane ring deformation vibrations or $\mathrm{C}-\mathrm{H}$ rocking is at $681 \mathrm{~cm}^{-1}$ which occurs at $695 \mathrm{~cm}^{-1}$ in our spectrum [18]. These peaks were observed in the present work for preparations using APS as oxidants and various dopants such $\mathrm{LiClO}_{4}, \mathrm{p}$-TS and NSA (Figure 1). This agrees well with the ones available the literature, confirming the formation of Polypyrrole [19,20].

\subsubsection{X-Ray Diffraction Analysis}

A typical $X$ ray diffraction pattern for polypyrrole prepared using APS as oxidant is shown in Figure 2.The XRD patterns for samples using the dopants also show broad peaks in the region $15^{\circ}<2 \theta<30^{\circ}$ revealing that the resulting polypyrrole powders are amorphous in nature. This agrees well with the structure reported in literature [14]. Such broad peak usually indicates short range arrangement of chains. The half width of this peak was measured for all the four samples and their values are given in Table 1. The half widths were measured from the diffraction curve from the angles higher than the peak positions and assuming Gaussian distribution for the low angle part of the diffraction curve.

However the diffraction peak centered at around $25.4^{\circ}$ for pure pyrrole shows some displacement when we go to the doped ones. In general the doping leads to shift the peaks toward the lower angle, being lowest for Ppy doped with p-TS $\left(24.6^{\circ}\right)$. This indicates that the inter planer spacing increases with the addition of dopants. This would make the chains get apart from each other due to the large sizes of the dopant molecules. In addition it was noted that the diffraction profile is highly asymmetrical and there is considerable broadening towards lower angles from the peak positions.

\subsubsection{Morphology Using SEM}

The morphological features of polypyrrole synthesized

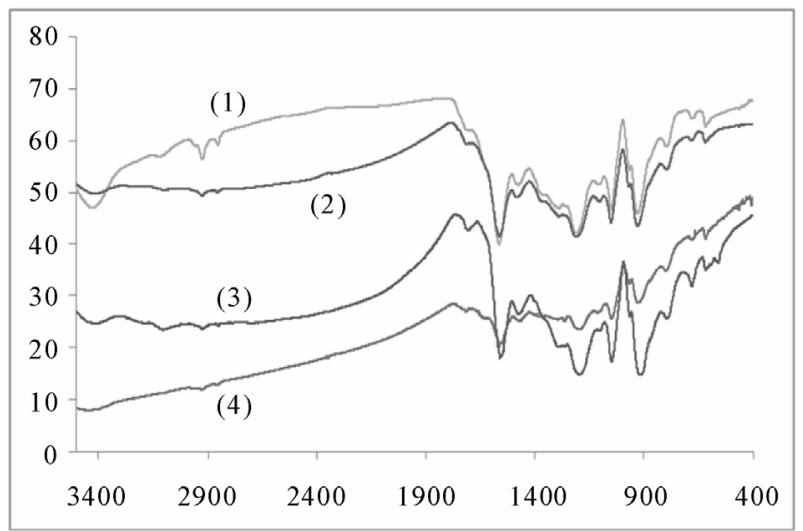

Figure 1. FTIR of polypyrrole using APS (2), doped with P-TS (1); NSA (3); $\mathrm{LiClO}_{4}$ (4).

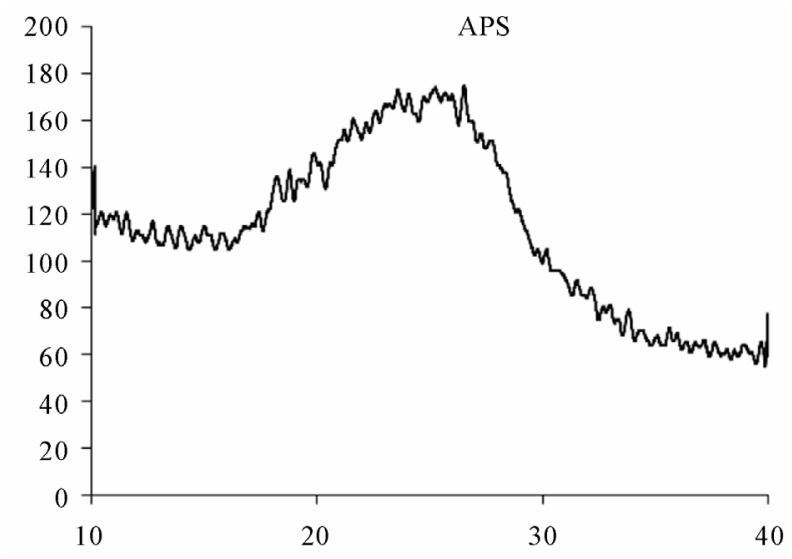

Figure 2. $\mathrm{X}$ ray diffraction pattern for polypyrrole prepared using APS as oxidant.

Table 1. X ray Peak Positions, Half widths, Globular sizes and conductivities of Ppy and with dopants.

\begin{tabular}{ccccc}
\hline Sample & X ray peak position & X ray half width & SEM size of globule & $\begin{array}{c}\text { Conductivity at room temp. } \\
\text { over a linear portion of curve }\end{array}$ \\
\hline Pure Polypyrrole & $25.4^{\circ}$ & $8^{\circ}$ & $0.59 \mu \mathrm{m}$ & $1.70 \times 10^{-3}$ \\
Polypyrrole with $\mathrm{LiClO}_{4}$ & $25.27^{\circ}$ & $3.24^{\circ}$ & $0.89 \mu \mathrm{m}$ & $1.02 \times 10^{-3}$ \\
Polypyrrole with p-TS & $24.6^{\circ}$ & $6.8^{\circ}$ & $0.27 \mu \mathrm{m}$ & $4.60 \times 10^{-3}$ \\
Polypyrrole with NSA & $25.3^{\circ}$ & $6.6^{\circ}$ & $0.63 \mu \mathrm{m}$ & $2.84 \times 10^{-3}$ \\
\hline
\end{tabular}


chemically and electrochemically has revealed that mostly the growth is in the globular form but changes some time due to dopant molecules [21]. Typical SEM images of different Polypyrrole preparations are shown in Figures 3 (a)-(d). All the photographs show a globular structure. It can be seen from Figure 3(a) that when polymerization was done with APS the average size of globules was found to be $0.59 \mu \mathrm{m}$. The individual granules observed were nearly spherical and have a close packing. It seems that such spherulites are growing one over the other and forming a continuous structure. The sizes of these spherulites are varying from $0.2 \mu \mathrm{m}$ to $0.7 \mu \mathrm{m}$. When dopants were used during the polymerization with APS, the sizes of the granules were found to be different. With $\mathrm{LiClO}_{4}$ the average size was found to be $0.89 \mu \mathrm{m}$, with variation between $0.3 \mu \mathrm{m}$ to $0.9 \mu \mathrm{m}$, which is rather large in comparison to other preparations. When p-TS were used as a dopant, there was a considerable reduction in the size amounting to $0.27 \mu \mathrm{m}$. More over the morphological feature was spongy in nature and there is considerable difficulty in distinguishing the granules from each other. This shows that a much closed packed structure is formed and this fact supports our earlier conclusion on the basis of X ray diffraction. When NSA was used as dopant it was observed that average globular size is 0.63 $\mu \mathrm{m}$. Such morphological features are considered to be good for gas sensing applications.

\subsection{I-V Characteristics}

Typical plots of I vs. V for polypyrrole prepared using APS as oxidant and $\mathrm{LiClO}_{4}$, p-TS and NSA as dopants are given in Figures 4(a)-(d). It was observed that the electrical conductivity of polypyrrole increased when dopants such as $\mathrm{LiClO}_{4}$, p-TS and NSA were used. Nearly linear relationship of the graph of I vs V curve was noted upto 8 volts for samples prepared using only APS and APS +

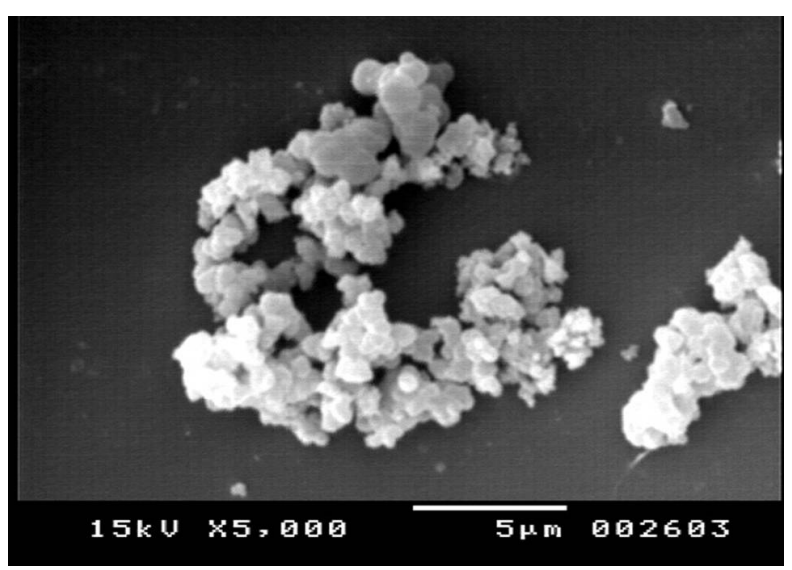

(a)

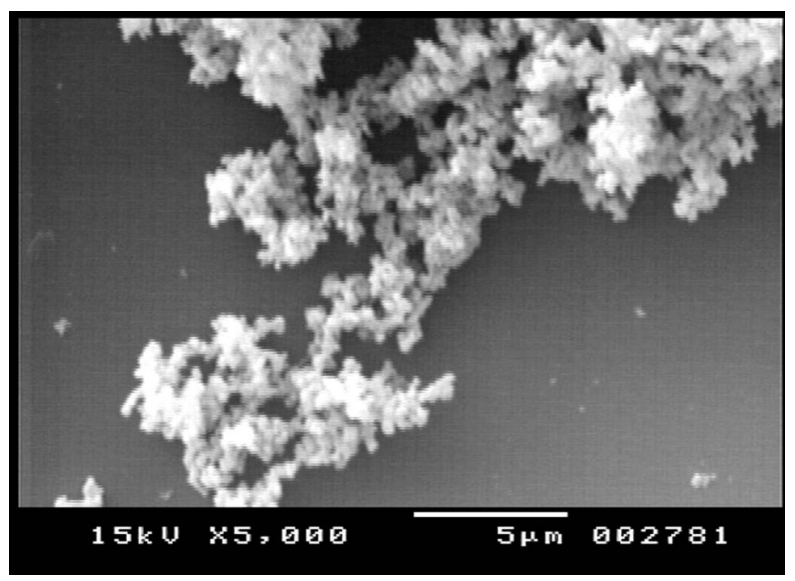

(c)

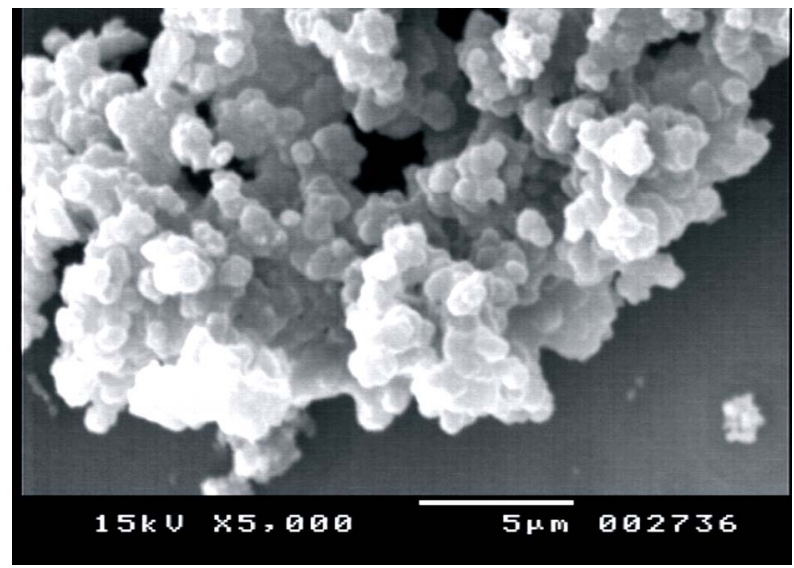

(b)

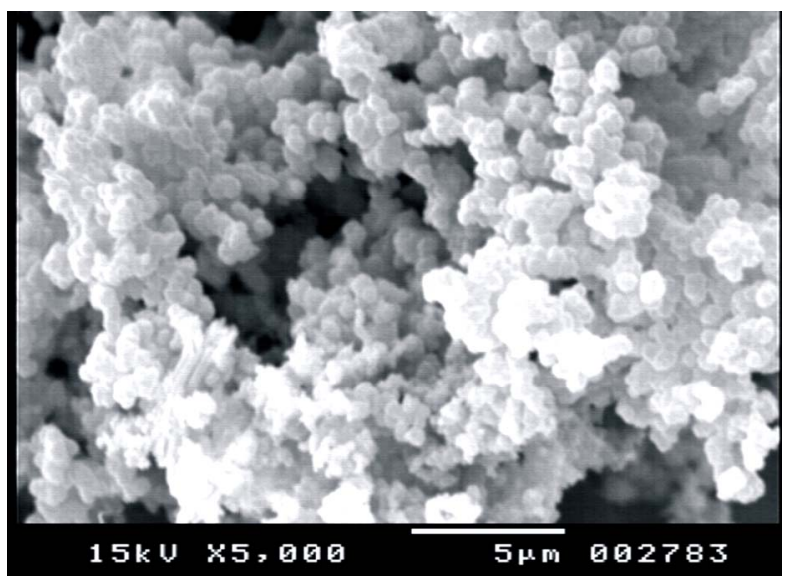

(d)

Figure 3. (a) SEM of Ppy prepared by our method using APS as an oxidant; (b) SEM of Ppy using APS as a oxidant and Li$\mathrm{ClO}_{4}$ as dopant prepared by our method; (c) SEM of Ppy using APS as an oxidant and p-Ts as dopant prepared by our method; (d) SEM of polypyrrole using APS as a oxidant and NSA as dopant prepared by our method. 


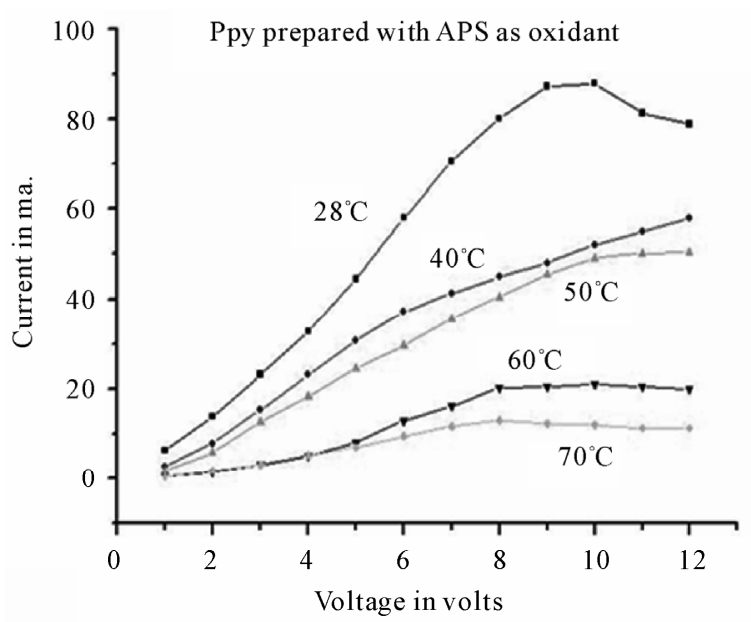

(a)

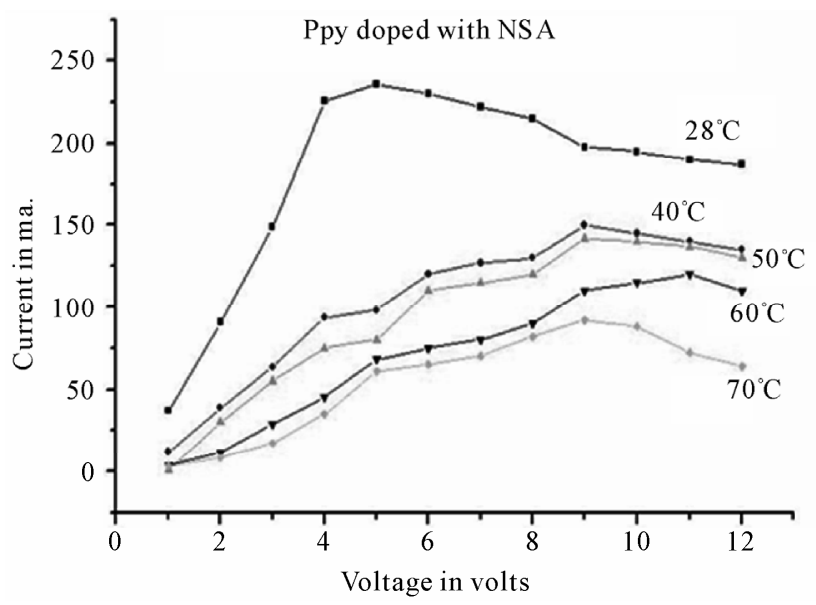

(c)

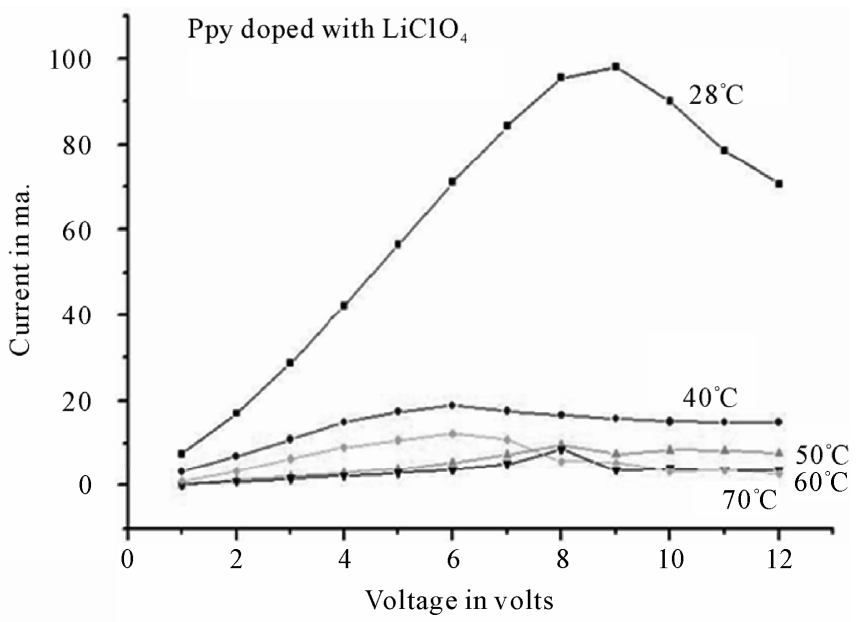

(b)

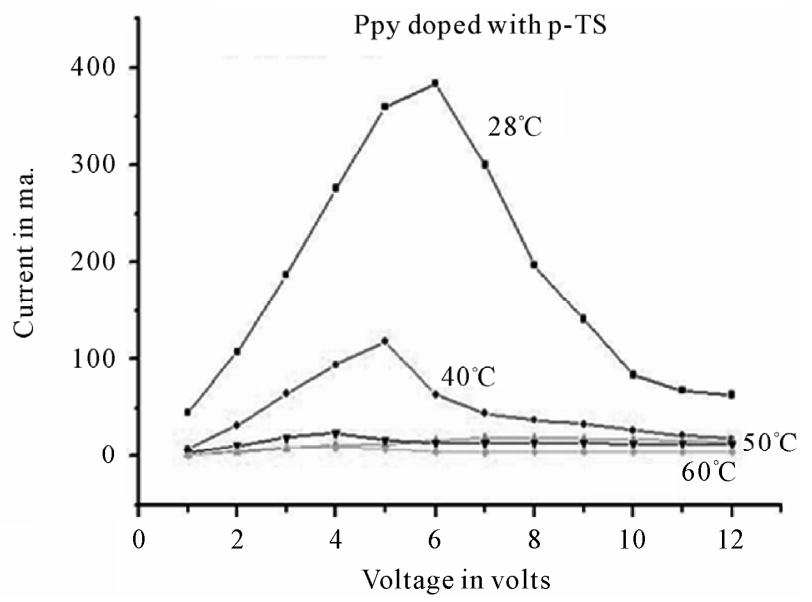

(d)

Figure 4. I vs V characteristic of Polypyrrole prepared using (a) APS; (b) $\mathrm{LiClO}_{4}$; (c) NSA; (d) p-TS.

$\mathrm{LiClO}_{4}$. When other dopants like NSA and p-TS were used the initial part of rising current peaked at 6 and 4 volts respectively. After this peaks when voltage was increased further, a decrease in current was observed. The reason for such departure from ohm's law is either that the current is mainly contributed by ions or degradation of the sample at higher voltage. However the latter reason can be ruled out as the temperature variation of the I vs $\mathrm{V}$ plot does not show such behavior of decreasing current at temperatures $40^{\circ} \mathrm{C}$ to $70^{\circ} \mathrm{C}$. Additional evidences for the contribution to the current by ions are being explored. The conductivities were found in the range of $1.0 \times 10^{-3}$ to $4.6 \times 10^{-3} \mathrm{~S} / \mathrm{cm}$.

The temperature dependence of the electrical conductivity of the polypyrrole, prepared with APS and their doped varieties were studied at various temperatures from $30^{\circ} \mathrm{C}$ up to $70^{\circ} \mathrm{C}$. It was observed that the electrical conductivity decreased gradually for all the samples.
This behavior resembles the metallic conductors. It may be mentioned that pure pyrrole is more like insulator but behaves like semiconductor in the doped form. The mechanism of conduction is supposed to be by polarons and bipolarons formation due to the dopant molecules [22].

The mechanism of polaron formation is illustrated in Figure 5. In case of polypyrrole the absence of electron in the chain leads to formation of holes i.e. p-type conduction. Thus addition of dopants leads to the modification of energy levels as shown in Figure 5.

Additional energy bands are formed above the valence band and just below the conduction band. This reduces the energy gap and the doping leads to semiconduction. Thus it is expected that increasing temperature will lead to increase of energy for an electron in the valance band which jumps to conduction band and hence the conductivity should increase with the temperature. However the 

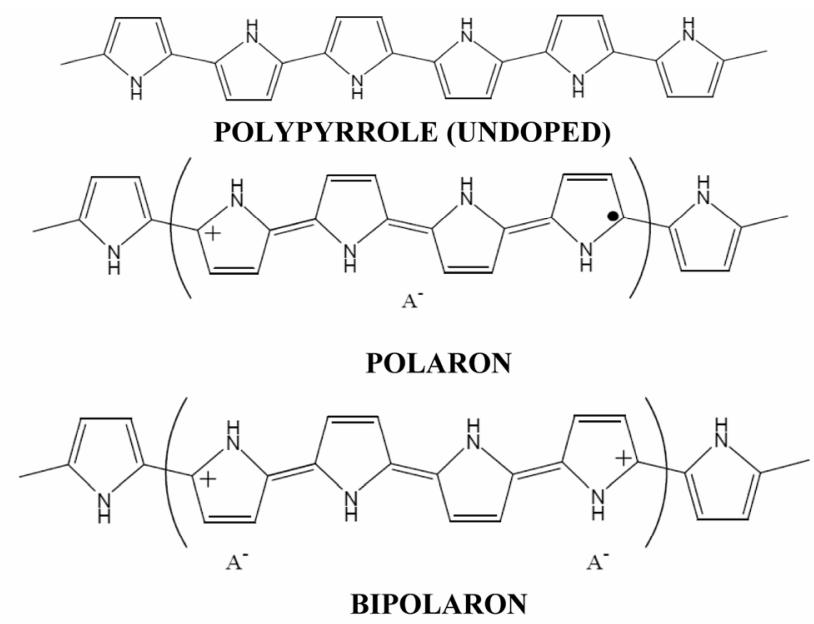

Conduction Band
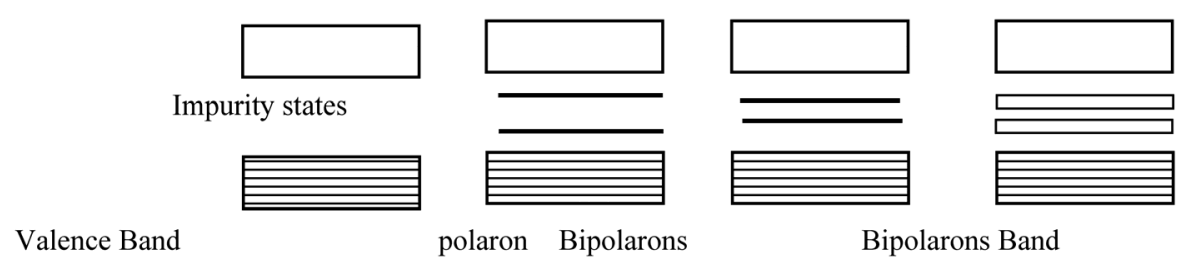

Bipolarons Band

Figure 5. Conduction mechanism in polypyrrole.

present studies revealed that the materials do not behave like semiconductor but like metals where the conductivity is decreasing with temperature. Such behavior can be caused due to the large number of intermediate energy states in the energy gap region. In the present studies the doping level was $10 \%$ which is comparatively high and leads to overlap of a large number of energy states. In addition the size of the dopants ions $\mathrm{ClO}_{4}$ and $\mathrm{SO}_{3}^{-}$is quite large and overlap of energy states can occur. Thus the temperature dependence can be understood. The conductivities change in the order $\mathrm{p}$-TS $>\mathrm{NSA}>\mathrm{LiClO}_{4}$ $>$ pure.

Of all the samples studied in the present investigation polypyrrole doped with p-TS was found to have maximum conductivity. In addition it was noted that the conductivity changes with temperature to a small extent for NSA and p-TS doped samples.

\subsection{Gas Sensor}

All the polypyrrole samples were studied for detection of ammonia gas. A typical plot of current vs time for polypyrrole prepared using APS as oxidant and exposed to ammonia gas is given in Figure 6. All samples were studied for 3 cycles to check their reproducibility and absorption and desorption process. It may be seen from the Figures 6(a)-(d) that the $\mathrm{i}$ vs $\mathrm{t}$ plot for $2^{\text {nd }}$ and $3^{\text {rd }}$ cycles somewhat differ from the first cycle. This may be because desorption may be not completed within the given time.

The Sensitivity factor is calculated using the equation

$$
\mathrm{S}=\frac{\mathrm{Rg}-\mathrm{Ro}}{\mathrm{Ro}}
$$

where $\mathrm{Rg}$ and Ro are resistances with gas and without gas (in air) respectively [23,24]. The values calculated during the present investigation for sensors fabricated using differently doped polypyrrole and for different gases are given in Table 2.

The response of different materials towards ammonia gas was seen to be different. When pure Ppy was exposed to ammonia gas there was an increase in current. This behavior is exactly opposite to that observed for Ppy prepared by electrochemical method [25]. This is mainly because in chemical preparation $\mathrm{S}_{2} \mathrm{O}_{8}^{2-}$ ion is incorporated as counter ion to maintain the electronutrality. Usually partial oxidation state (upto +0.33 ) is produced in the process. Thus there is a possibility that higher oxidation state can be induced in the interaction with ammonia. In such a situation the higher charge on cations can lead to increase in current.

It was noted during these investigations that when Ppy doped with $\mathrm{LiClO}_{4}$, p-TS and NSA were used, a decrease in current was observed when exposed to ammonia gas. The electrical conductivity of these products showed 


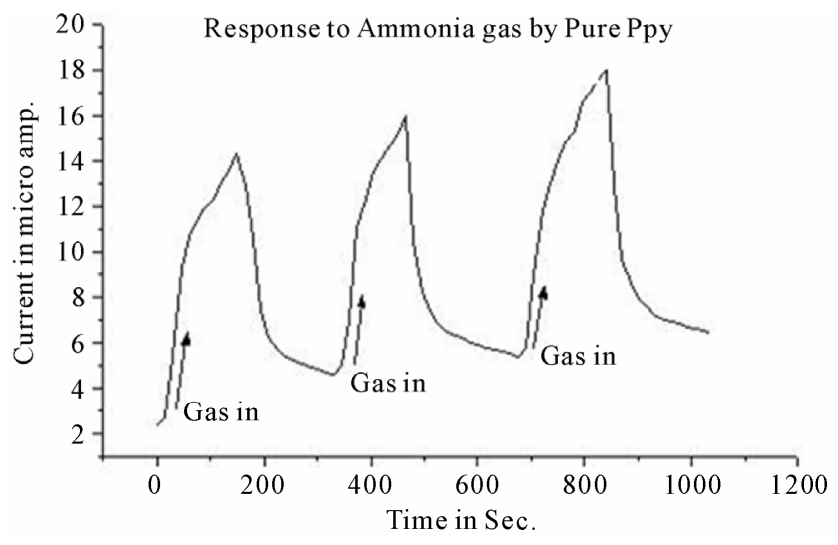

(a)

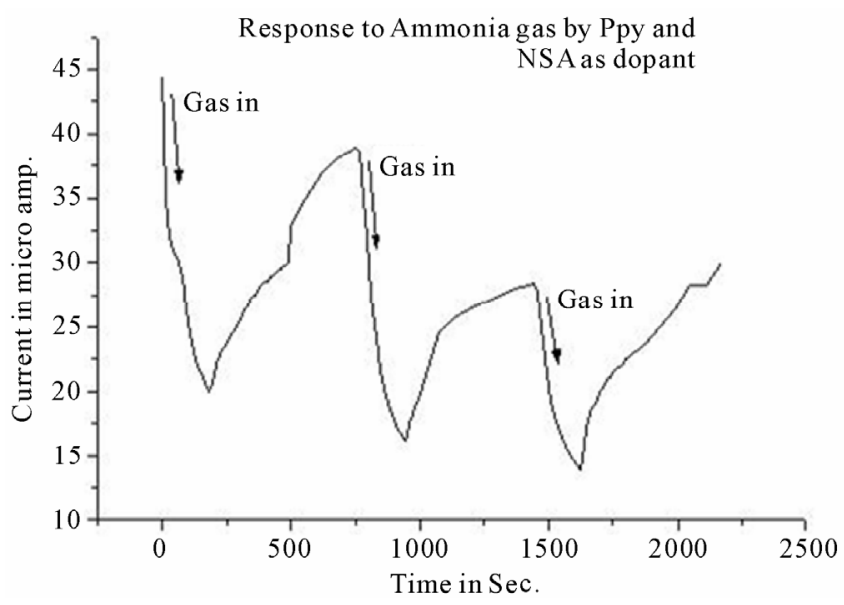

(c)

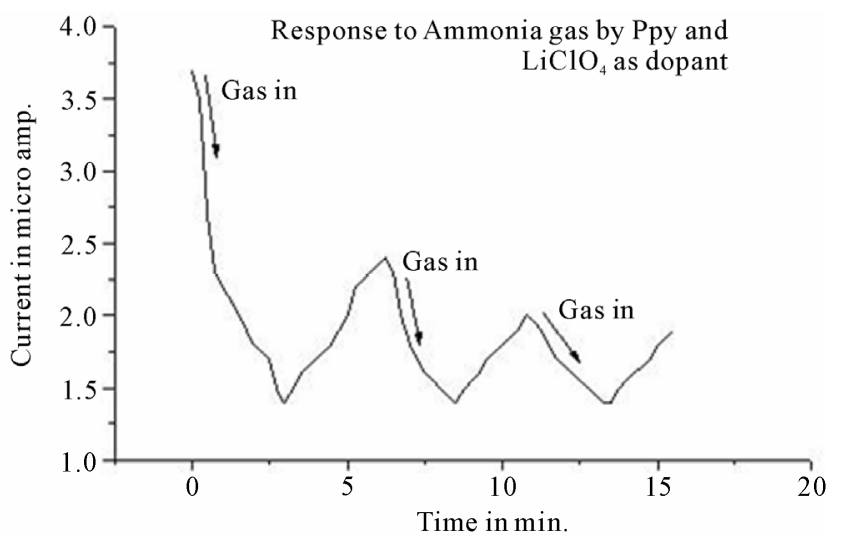

(b)

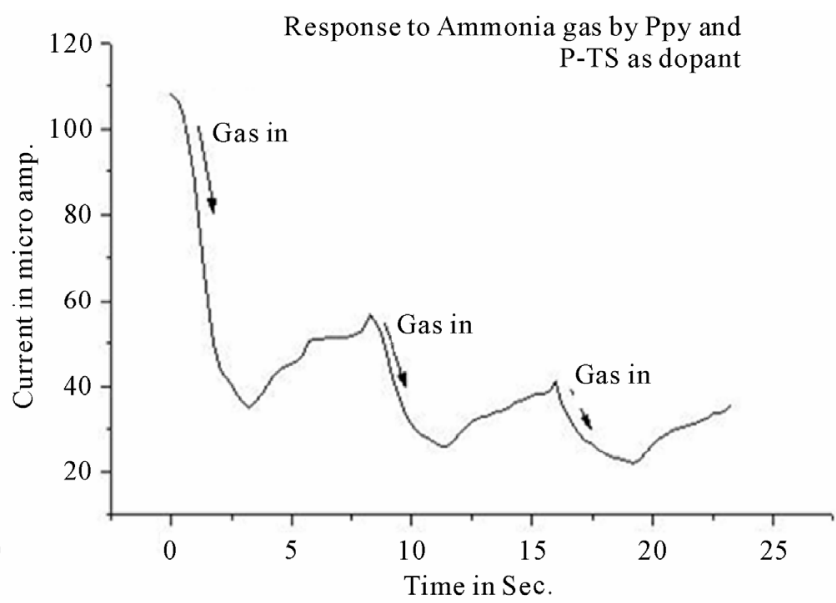

(d)

Figure 6. Response for ammonia of polypyrrole prepared using (a) APS; (b) $\mathrm{LiClO}_{4}$; (c) NSA; (d) p-TS.

Table 2. Sensitivity of pure and doped Ppy for ammonia gas.

\begin{tabular}{cc}
\hline Sample & Sensitivity for Ammonia \\
\hline Pure Polypyrrole & 0.54 \\
Polypyrrole with $\mathrm{LiClO}_{4}$ & 1.28 \\
Polypyrrole with p-TS & 2.08 \\
Polypyrrole with NSA & 2.86 \\
\hline
\end{tabular}

higher conductivity than pure Ppy which means that the doping levels and the number of charge carriers produced in the process are quite high. This is probably due to the presence of sulfonic acid group inducing more charge on pyrrole ring. Thus there already exists a higher density of charge and therefore the presence of ammonia cannot produce more charges, but rather decreases the effective charge. In such a situation therefore the conductivity of Ppy will decrease, which has indeed been observed in our studies. It may be noted from the curves $b$ to $d$ that the variations in the current in the second and third cycles decreases, which is probably due to the fact that, the desorption of the gas is not complete in the time interval that was allowed in the present investigation.

\section{Conclusions}

Polypyrrole was synthesized in the pure form and doped with $\mathrm{LiClO}_{4}$, p-TS and NSA. All these four verities were characterized using FTIR, $X$ ray diffraction and SEM. It was seen that there are structural and morphological differences which affect their electrical properties. The electrical conductivity was highest for $\mathrm{p}$-TS doped polypyrrole $\left(4.6 \times 10^{-3} \mathrm{~S} / \mathrm{cm}\right)$. These materials were used as gas sensors for the detection of ammonia. It was found that ammonia could be detected efficiently.

\section{Acknowledgements}

The authors wish to thank Director, BTRA for interest and support during this work. Thank are also due to Mr. 
V. E. Walunj for help during the experimental work. H. K. C. is grateful to the Principal Dnyanasadhana College for granting the necessary leave.

\section{REFERENCES}

[1] S. C. Hernandez, D. Chaudhari, W. Chen, N. Myung and A. Mulchandani, "Single Polypyrrole Nanowire Ammonia Gas Sensor," Inter Science, Vol. 19, No. 19-20, 2007, pp. 2125-2130.

[2] S. Cosnier, C. Gondran, R. Wessel, F.-T. Montforts and M. Wedel, "A Poly(Pyrrole-Cobalt (II) Deuteroporphyrin) Electrode for the Potentiometric Determination of Nitrite," Sensors, Vol. 3, No. 7, 2003, pp. 213-222. doi:10.3390/s30700213

[3] M. F. Mabrook, C. Pearson and M. C. Petty, "InkjetPrinted Polypyrrole Thin Films for Vapor Sensing," Sensors and Actuators B, Vol. 115, No. 1, 2006, pp. 547-557. doi:10.1016/j.snb.2005.10.019

[4] H. Bai and G. Shi, "Gas Sensors on Conducting Polymers," Sensors, Vol. 7, No. 3, 2007, pp. 267-307. doi: $10.3390 / \mathrm{s} 7030267$

[5] R. A. Bissell, K. C. Persaud and P. Travers, "The Influence of Non Specific Molecular Partitioning of Analytes on the Electrical Response of Conducting Organic Polymer Gas Sensors," Physical Chemistry Chemical Physics, Vol. 4, No. 14, 2002, pp. 3482-3490. doi: $10.1039 / \mathrm{b} 201292 \mathrm{~h}$

[6] S. C. Hernandez, D. Chaudhuri, W. Chen, N. V. Myung and A. Mulchandani, "Single Polypyrrole Nanowire Ammonia Gas Sensor," Electroanalysis, Vol. 19, No. 19-20, 2007, pp. 2125-2130.

[7] C. Nylabder, M. Armgrath and I. Lundstrom, "An Ammonia Detector Based on a Conducting Polymer," Proceedings of the International Meeting on Chemical Sensors, Fukuoka, 19-22 September 1983, pp. 203-207.

[8] H. Bai and G. Q. Shi, "Gas Sensors Based on Conducting Polymers," Sensors, Vol. 7, No. 3, 2007, pp. 267-307.

[9] Q. Fang, D. G. Chetwynd and J. W. Gardner, "Conducting Polymer Films by UV-Photo Processing," Sensors and Actuators A, Vol. 99, No. 1-2, 2002, pp. 74-77. doi:10.1016/S0924-4247(01)00894-9

[10] L. Ai Mashat, H. D. Tran, W. Wlodarski, R. B. Kaner and K. K. Zadeh, "Conductometric Hydrogen Gas Sensors Based on Polypyrrole Nanofibers," Sensors Journal, Vol. 4, No. 4, 2008, pp. 365-370.

[11] A. Joshi, S. A. Gangal, N. Padma, D. K. Aswal and S. K. Gupta, "Gas Sensing Properties of Polypyrrole Thin Films," BARC Newsletter, No. 297, 2008, pp. 236-239.

[12] S. Hosein and A. A. Entezami, "Polypyrrole Based Toxic Gas Sensors by Mass and Conductivity Measurements," Iranian Polymer Journal, Vol. 8, No. 3, 1999, pp. 205213.

[13] S. S. Jeon, H. H. An, C. S. Yoon and S. S. Im, "Synthesis of Ultra-Thin Polypyrrole Nanosheets for Chemical Sen- sor Applications," Polymer, Vol. 52, No. 3, 2011, pp. 652-657. doi:10.1016/j.polymer.2010.12.021

[14] G. Harsanyi, "Highly Sensitive and Selective Ammonia Sensor for Environmental Monitoring and Workplace Safety Applications".

[15] H. Yoon, M. Chang and J. Jang, "Sensing Behavior of Polypyrrole Nano Tubes Prepared in Reverse Micromulsions: Effects of Tranducers Size and Transduction Mechanism," Journal of Physical Chemistry B, Vol. 110, No. 29, 2006, pp. 14074-14077. doi:10.1021/jp061423b

[16] V. Saxena, S. Choudhary, S. C. Gadkari, S. K. Gupta and J. V. Yakhmi, "Room Temperature Operated Ammonia Gas Sensor Using Polycarbazole Langumuir-Blodgett Film," Sensors and Actuators B, Vol. 107, No. 1, 2005, pp. 277-282. doi:10.1016/j.snb.2004.10.011

[17] L. Jiang, H. K. Jun, Y. S. Hoh, J. O. Lim, D. D. Lee and J. S. Huh, "Sensing Characteristics of Polypyrrole-Poly (Vinyl Alcohol) Methanol Sensors Prepared by in Situ Vapour State Polymerization," Sensors and Actuators B Vol. 105, No. 2, 2005, pp. 132-137. doi:10.1016/j.snb.2003.12.077

[18] R. Ansari, "Polypyrrole Conducting Electroactive Polymers: Synthesis and Stability Studies," E-Journal Chemistry, Vol. 3, No. 13, 2006, pp. 186-201.

[19] H. Kato, O. Nishikwa, T. Matsui, S. Honma and H. Kokado, "Fourier Transform Infrared Spectroscopy Study of Conducting Polymer Polypyrrole Higher Order Structure of Electrochemically Synthesized Film," The Journal of Physical Chemistry, Vol. 95, No. 15, 1991, pp. 6014 6016. doi:10.1021/j100168a055

[20] N. V. Bhat, A. P. Gadre and V. A. Bambole, "Structural, Mechanical and Electrical Properties of Elctro-Polymerized Polypyrrole Composite Films," Journal of Applied Polymer Science, Vol. 80, No. 13, 2001, pp. 2511-2517. doi:10.1002/app.1359

[21] T. K. Vishnuvardhan, V. R. Kulkarni, C. Basavaraja and S. C. Raghavendra, "Synthesis, Characterization and A.C. Conductivity of Polypyrrole/ $\mathrm{Y}_{2} \mathrm{O}_{3}$ Composites," Material Science, Vol. 29, No. 1, 2006, pp. 77-83.

[22] H. Eisazadeh, "Studying the Characteristics of Polypyrrole and Its Composites," World Journal of Chemistry Vol. 2, No. 2, 2007, pp. 67-74.

[23] R. Turcu, M. Brie, G. Leising, V. Tosa, A. Mihut, A. Niko and A. Bot, " FTIR Reflectance Studies of Electrochemically Prepared Polypyrrole Films," Applied Physics $A$, Vol. 67, No. 3, 1998, pp. 283-287. doi:10.1007/s003390050772

[24] S. A. Waghule, S. M. Yenorkar, S. S. Yawale and S. P. Yawale, "SnO 2 /Ppy Screen-Printed Multilayer $\mathrm{CO}_{2}$ Gas Sensor," Sensors and Transducers Journal, Vol. 79, No. 5, 2007, pp. 1180-1185.

[25] R. Naillathambi, "Preparation and Characterization of Conducting Polymers and Its Composites for Gas Sensors,' PhD Dissertation, University of Bombay, Mumbai, 1996. 